\title{
Vibration Frequency Adaptive Control of the Flexible Sampling Robot based on ANFIS
}

\author{
Wei Lu ${ }^{1 *}$, Yun Ling ${ }^{2}$, Ai-guo Song ${ }^{2}$, Wei-min Ding ${ }^{1}$, Xian-lin Zhao ${ }^{1}$ and Hong Zeng ${ }^{2}$ \\ ${ }^{1}$ College of Engineering, Nanjing Agricultural University/Jiangsu Province \\ Engineering Lab for Modern Facility Agriculture Technology \& Equipment, Nanjing \\ Agricultural University, Nanjing 210031, China \\ ${ }^{2}$ School of Instrument Science and Engineering, Southeast University/ Key \\ Laboratory of Remote Measuring and Control in Jiangsu Province, Nanjing 210096, \\ China \\ *njaurobot@njau.edu.cn
}

\begin{abstract}
Comparing to the big volume, large weight and high power consumption of the conventional samplers which are fixed on the lunar rover, the paper firstly described a novel flexible mini lunar sampling robot. Then the nonlinear dynamics resonance broken system is built to model the contact between the sampling robot and the lunar regolith. It is found to be suitable for drilling when the sampling robot is in the resonance condition. For the nonlinear time-varying system of the dynamic modeling of the sampler in drilling, we presented the method of the frequency neural-fuzzy adaptive control based on the dynamic resonant frequency prediction of the flexible sampling robot using neural networks. Firstly the algorithm predicts the dynamic resonant frequency of the sampling robot by GRNN. Then a neural-fuzzy adaptive control system is established, in which the frequency prediction error, the amplitude and its variable are adopted as the input and the sweep frequency bandwidth as the output, to adjust the frequency bandwidth dynamically. What's more, the simulation results verify the effectiveness of the control strategy. Finally, the experimental results show that the control algorithm can improve the drilling depth, drilling efficiency and the discarding efficiency by $66.7 \%, 65.2 \%$ and $67.4 \%$, respectively, in stimulant lunar regolith.
\end{abstract}

Keywords: Flexible robot; GRNN; ANFIS; Adaptive control

\section{Introduction}

Lunar exploration is of great economic efficiency and scientific significance, and it is very hot in the worldwide nowadays [1]. The tele-operation of the rover exploring on the lunar surface is an important technology that normally used for sampling and analyzing the lunar soil. But the lunar rover gets its power from solar panels and rechargeable batteries which could not initially provide high-power energy. So in the process of designing the lunar sampler, the low power consumption, small volume and low weight should be considered.

Till now, numerous researchers have studied and developed many samplers for planetary exploration. All planet samplers can be divided into two types: the rigid structure and the flexible structure. The former type samplers are the samplers used in Luna 16 (1970) and Luna 20 (1972) of Soviet is single-rod [2], the lunar regolith sampler used in Surveyor 5 (1967), the Instrument Deployment Device (IDD) of 
NASA's Mars Exploration Rovers (2003) and the JPL's Rover with tandem joint type structure arms which have more freedom [3, 4]. They have characteristics such as excellent stiffness and flexural capacity but too heavy weight and big contraction volume. The latter type, such as the arms of Mole-typed Deep Sampling Robot (ESA in 2003[5], Japan in 2005[6]) and the Earthworm-type samplers [7] from Japan (2007) which have little hardness, cannot guide well and can only be used for once. The structure of the deployable wood wasp drill is an elastic structure which has many advantages such as light weight, low power consumption but the disadvantages also exist, i.e., the low sampling efficiency and small sampling volume $(2006)$ [8,9].

In order to overcome the disadvantages of the existing samplers, we have developed a novel flexible lunar sampling robot which has the advantages of small shrink volume, long working distance, light weight, low power consumption, and well adaptivity.

As we all known, the flexible sampler can be controlled always in resonance state for improving the drilling efficiency. But the dynamical modeling of the sampler-regolith system is difficult to realize and it varies along with time when the sampler is in different depth in the lunar regolith. As far as we know, the existing control methods of vibration are mostly used in order to reduce the vibration amplitude [10-13]. Besides that, the vibration frequency is about dozens $\mathrm{kHz}$ which is far from that of the flexible sampler whose resonant frequency is about several to dozens of Hertz [14, 15].

This paper focuses on the control of the flexible sampling robot, namely, always keep it in the resonance state while drilling by adaptive frequency control to improve the efficiency. The paper is organized as follows. The next section introduces the mechanical design of the novel flexible mini lunar regolith sampling robot. Then the nonlinear dynamics resonance broken system of "sampling head-lunar regolith" is established and discussed in section3. Section 4 presents the method of the frequency ANFIS control based on the dynamic resonant frequency prediction of the flexible sampling robot by GRNN (Generalized Regression Neural Network). In Section 5, the sampling experiments are carried out and discussed. Finally, some conclusions are drawn in Section 6.

\section{Mechanical Design of the Flexible Lunar Regolith Sampling Robot}

A novel sampling robot is developed which mainly includes four parts: a box for retractable tape spring, open cylindrical shell, driving structure and a sampling head (see Figure 1).

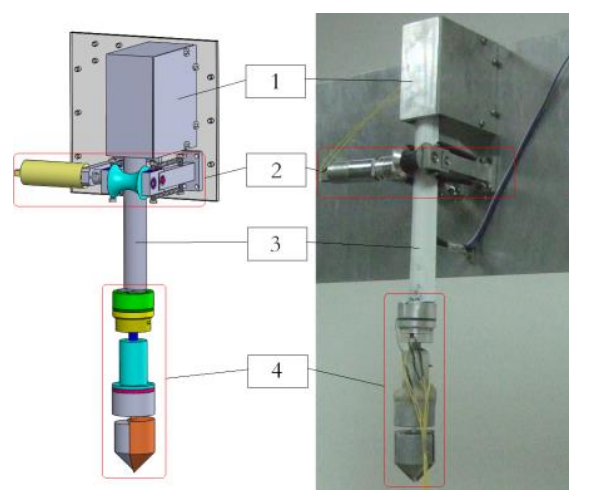

[1] Box for retractable tape spring [2] Driving structure [3] Open cylindrical shell i.e. sampling arm [4] Sampling head

Figure 1. Structure of the sampling robot 
The cylindrical shell is gripped by the convex and concave rollers which shapes match well with that of the cylindrical shell. The surfaces of the convex roller and the concave roller are covered with soft metal (or soft rubber) to ensure the cylindrical shell can get the maximum moment [16]. The concave roller is connected to the slots in both sides by bearings which are fixed in the brackets by strings. The convex roller acting as friction wheel is fixed in the brackets by slots and connected to the motor by a coupling. The friction transmits the movement to the open cylindrical shell when the motor rotates. So the sampling arm moves down when the motor rotates counter clockwise, and vice versa. Only one $3 \mathrm{~W}$ motor is used to achieve the extension and contraction of the sampling arm, so the power consumption of the arm is very low.

Figure 2 shows the mechanism of the sampling head. A $0.5 \mathrm{~W}$ longitudinal vibrator and a $0.3 \mathrm{~W}$ torsion vibrator whose frequencies can be adjusted are embedded between a lid and an acoustic horn from above to below in order. A $1 \mathrm{~W}$ sampling motor is fixed to the enclosure and it can drive the sampling spoons to rotate in different directions at the same speed by gears, so the sampling head has no output torque when it is working. Besides, the sampling head is characterized by small volume, light weight, low power consumption and it can sample a full scoop of about $5 \mathrm{~cm} 3$ simply by rotating the spoon one circle, hence it can be used in the in-situ sampling of shallow lunar regolith quantitatively.



[1] lid [2] longitudinal vibrator [3] torsional vibrator [4] transducer horn [5] sampling motor [6] gears [7] external sampling spoon [8] internal sampling spoon

Figure 2. Lunar regolith sampling head

\section{Dynamic Resonant Broken System}

The contact between the sampling head and lunar regolith can be considered as a rigid-powder coupling system. The sampling head's circumference unit can be equivalent to a unilateral shock vibration broken system as shown in Figure 3. 


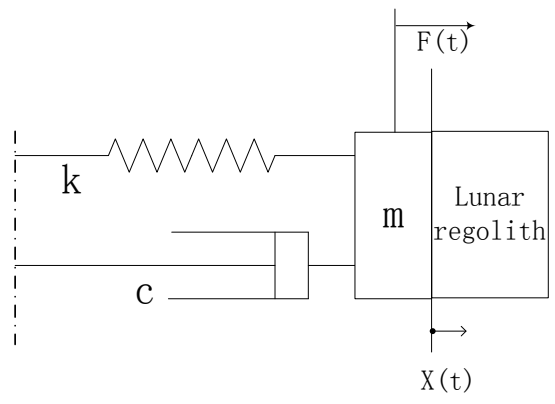

Figure 3. Unilateral shock vibration broken system

According to the vibration theory, the vibration equation Eq.(1) of the system can be given when an incentive signal $F(t)=k A \sin (\omega t)$ is exerted to the sampling head.

$$
m(t)+c x(t)+k x(t)=k A \sin (\omega t)
$$

Let $c=2 m \omega_{n} \zeta, \omega_{n}=\sqrt{k / m}, \beta=\omega / \omega_{n}$, where $m$ is the effective mass of the sampler head, $\omega$ is the frequency of the external excitation, $\omega_{n}$ is the resonance frequency of the vibration system; $A$ is the amplitude of the external excitation; $\zeta$ is the damping ratio; $k$ is the equivalent stiffness of the lunar soil and sampling arm which directly determines the resonant frequency.

The formula (1) can be transformed into the normalized form as follows:

$$
\frac{c}{m} \frac{k}{m} x+\frac{1}{m} f(x)=\frac{B}{m} \sin (\omega t)
$$

The fourth order Runge-Kutta method was adopted for simulation and calculation of the equation (2) because the numerical solution method is commonly used for complicated nonlinear dynamic model.

Firstly, the second-order vibration equations can be rewritten as one order state-space equations. The state variables are introduced as:

$$
\left\{\begin{array}{l}
y_{1}=x \\
y_{2}=\&
\end{array}\right.
$$

The formula (2) can be rewritten as below:

$$
\left\{\begin{array}{l}
\&=y_{2} \\
\&=-\frac{c}{m} \& \frac{k}{m} x-\frac{1}{m} f(x)+\frac{B}{m} \sin (\omega t)
\end{array}\right.
$$

The solution of the state equation can be obtained as follows:

$$
\left\{\begin{array}{l}
y_{1}(i+1)=y_{1}(i)+\frac{h}{6}\left(K_{1}+2 K_{2}+2 K_{3}+K_{4}\right) \\
y_{2}(i+1)=y_{2}(i)+\frac{h}{6}\left(L_{1}+2 L_{2}+2 L_{3}+L_{4}\right)
\end{array}\right.
$$


And the parameters are as follows:

$$
\left\{\begin{array}{l}
K_{1}=y_{2} \\
K_{2}=y_{2}+\frac{h}{2} L_{1} \\
K_{3}=y_{2}+\frac{h}{2} L_{2} \\
K_{4}=y_{2}+h L_{3} \\
L_{1}=-\frac{c}{m} y_{2}-\frac{k}{m} y_{1}-\frac{1}{m} f\left(y_{1}\right)+\frac{B}{m} \sin (\omega h k) \\
L_{2}=-\frac{c}{m}\left(y_{2}+\frac{h}{2} L_{1}\right)-\frac{k}{m}\left(y_{1}+\frac{h}{2} K_{1}\right)-\frac{1}{m} f\left(y_{1}+\frac{h}{2} K_{1}\right)+\frac{B}{m} \sin (\omega h k) \\
L_{3}=-\frac{c}{m}\left(y_{2}+\frac{h}{2} L_{2}\right)-\frac{k}{m}\left(y_{1}+\frac{h}{2} K_{2}\right)-\frac{1}{m} f\left(y_{1}+\frac{h}{2} K_{2}\right)+\frac{B}{m} \sin (\omega h k) \\
L_{4}=-\frac{c}{m}\left(y_{2}+h L_{3}\right)-\frac{k}{m}\left(y_{1}+h K_{3}\right)-\frac{1}{m} f\left(y_{1}+h K_{3}\right)+\frac{B}{m} \sin (\omega h k)
\end{array}\right.
$$

The parameters of the sampling head are: $m_{0}=0.4 \mathrm{~g}, e=2 \mathrm{~mm}, m=120 \mathrm{~g}, \omega_{0}=400 \pi$, $B=1.26 \sin (1256 t)$. When the length of the sampling arm is $80 \mathrm{~cm}$, the parameters can be obtained as $k=12 \mathrm{~N} / \mathrm{m}, \zeta=0.05, \omega=6.28 \sim 219.8, c=0.012, \omega=0.075 \sim 2.64$.

Through the numerical calculation with fourth order Runge-Kutta method, the curves of the displacement and velocity vary with time, the phase between the displacement and the velocity, the frequency spectrum graph of the displacement were obtained as shown in Figure 4.

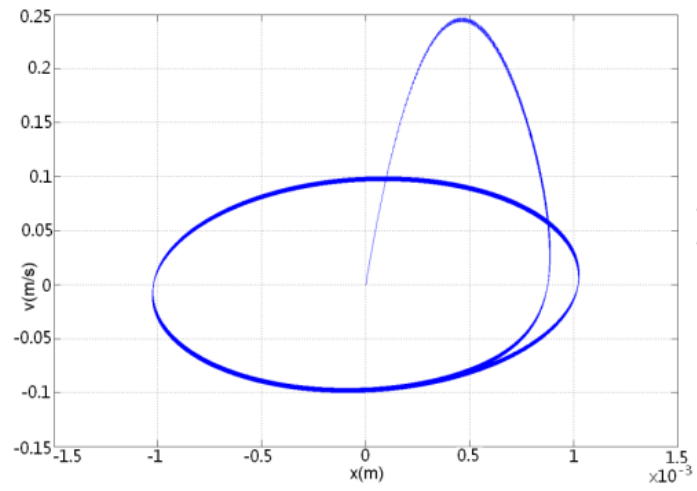

(a) Phase-diagram

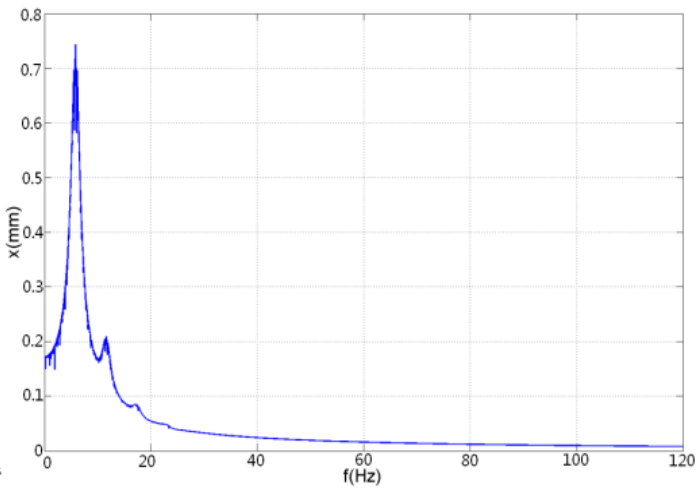

(b) Displacement- spectrum

Figure 4. Numerical simulation results

Simulation results show that the curves of displacement and velocity are generally symmetrical sinusoidal waves when the sampling head vibrates steadily. The vibration amplitude is maximal in the resonance state of the system. And in this situation, the lunar regolith contacting with the sampling head achieves the maximum amplitude, the internal friction angle value is decreased, the bond force is reduced and fluidity is enhanced. 


\section{Frequency Neural-Fuzzy Adaptive Control System Based on the Dynamic Prediction by GRNN}

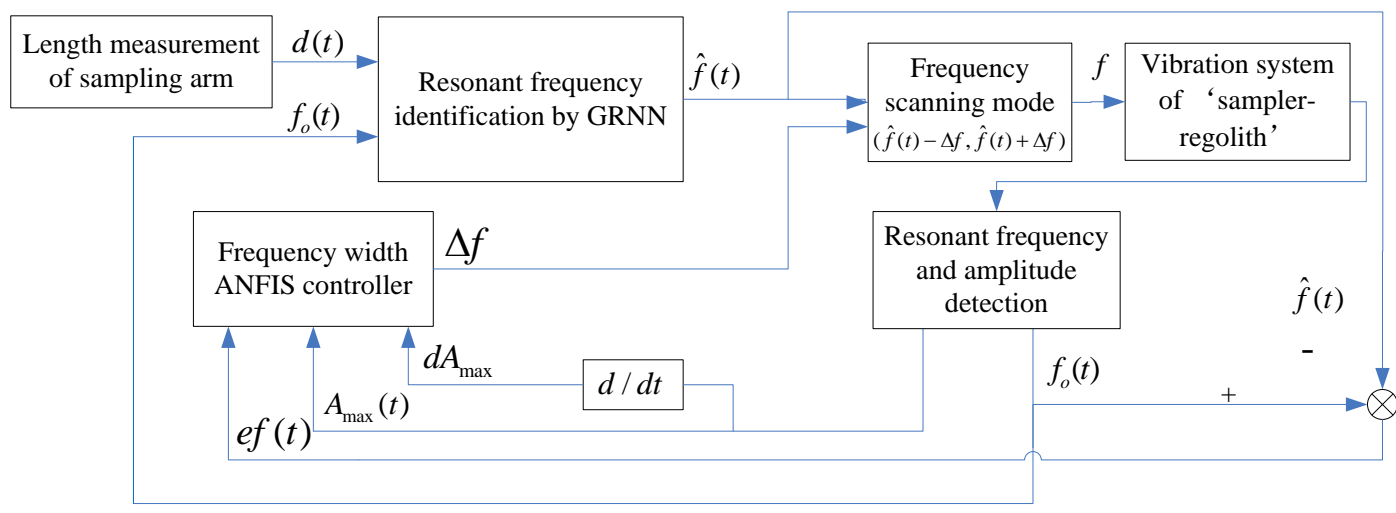

Figure 5. The ANFIS frequency control scheme based on the dynamic
prediction by GRNN

In practice, it's difficult to establish an accurate dynamic model of the sampling head contacting with regolith while drilling in different depth in an unknown environment of the lunar surface considering the electrical wires and the nonlinear damping varies with the depth and etc. So the only way to detect the resonant frequency of it is swept excitation. And the frequency corresponding to the maximum amplitude is the resonant frequency. But it is inefficient if the sweeping frequency band is broad. Therefore, the GRNN neural network is applied to predict the resonant frequency of the sampling head-regolith vibration system because it was aproved to be an excellent parallel datahanding method with dark-box operating performance, powerful studying and generalizing ability. Simultaneously, the fuzzy control algorithm is used to control the vibration frequency with the inputs such as frequency prediction error, vibratiing amplitude and its variation. And the adaptive neuro-fuzzy intefrence system (ANFIS) is used to bulid the fuzzy rules due to that the fuzzy control rules increase significantly with more input variables.

The control system is shown in Figure 5, which mainly consists of two parts: dynamic resonant prediction by GRNN and frequency adaptive control by ANFIS controller.

The performance index function of the system is as follows:

$$
J=E\left\{(e f(t))^{2}\right\}=E\left(f_{o}(t)-\hat{f}(t)\right)^{2}=\operatorname{Min}
$$

\subsection{The dynamic prediction of resonant frequency based on GRNN}

GRNN neural network was first proposed by Donald F. Specht in 1991. It is constructed based on mathematical statistics which is a type of radial basis function neural network (RBFNN). It does not require the pre-determined form of equations, instead, it uses the probability density function, so that it has a strong nonlinear mapping ability, flexible network structure, a high degree of error tolerance and robustness. Even if a small number of sample points exist, the output of the network can converge to the optimal regression surface, which is quite suitable for solving nonlinear 
problems [17]. Therefore, the GRNN neural network was selected to predict the dynamic resonant frequency of the sampling head-regolith system.

The structure of GRNN is very similar to RBF (Radial Basis Function) neural network which contains four layers: input layer, pattern layer, summation layer and output layer as shown in Figure 6 . The input of the network is $D=[d]$, and the output is $F=[f]$.

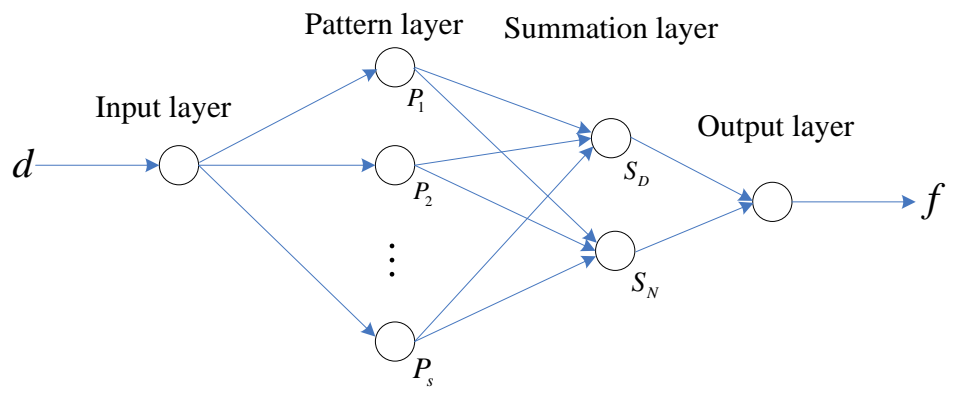

Figure 6. Neural network configuration of GRNN for resonant frequency prediction

Layer 1 -input layer: the neuron at layer 1 is input neuron that delivers input various elements of the input vector to the pattern layer. In this paper, the input dimension is only 1 which is the depth of the sampling head drilling.

Layer 2-pattern layer: The number of neurons in pattern layer is equal to that of learning samples. Each neuron corresponds to different sample. The transfer fuction of neuron $i$ of pattern layer is

$$
P_{i}=\left[\frac{\left(x-x_{i}\right)^{T}\left(x-x_{i}\right)}{2 \sigma^{2}}\right], i=1,2, \ldots, m
$$

Where $x$ is the input variables of the network; $x_{i}$ is the learning sample corresponding to the neuron $i ; \sigma$ is the smoothing parameter.

Layer 3-summation layer: the summation layer consists of 2 types of neurons. One does an arithmetic summation of the output of all neurons in pattern layer. The connectional weight values of the neuron and all the neurons in pattern layer are 1 . The transfer function of the neuron is as follows:

$$
S_{D}=\sum_{i=1}^{n} P_{i}, j=1,2, \ldots, s
$$

The other one does a weighted summation of the output of all neurons in pattern layer. The connectional weight value of the ith neuronin pattern layer and the jth neruon is the jth element $y_{i j}$ in the ith sample $y_{i}$. The transfer function of the jth neuron of summation layer is as folllows:

$$
S_{j}=\sum_{i=1}^{n} y_{i j} P_{i}, j=1,2, \ldots, s
$$

Layer 4-output layer: the number of neurons of the output layer is equal to the 
dimension $s$ of the output variables of learning samples. The output value of the $\mathrm{jth}$ neuron of the output layer results is shown as below:

$$
y_{j}=\frac{S_{j}}{S_{D}}, j=1,2, \ldots, s
$$

The initialization is a learning process of the training sample in GRNN. The network structure and connection weights between neurons will be determined if the learning samples are determined. The training of the network is actually a process of determining the smooth factors to adjust the transfer function of each unit to get the best regression estimate results. The steps to determine the optimal values of smooth factors are as follows:

Step 1: Let the smooth factor incrementally increase within a certain range of value.

Step 2: Remove 2 samples and train the neutron network using the rest samples, then test the network by these 2 samples.

Step 3: Calculate the absolute error values of the testing samples using the trained neutron network.

Step 4: Repeat step 2 and step 3 until all the training samples are used for testing not less than 1 time, then obtain the average prediction error and let it to be the optimal objective function.

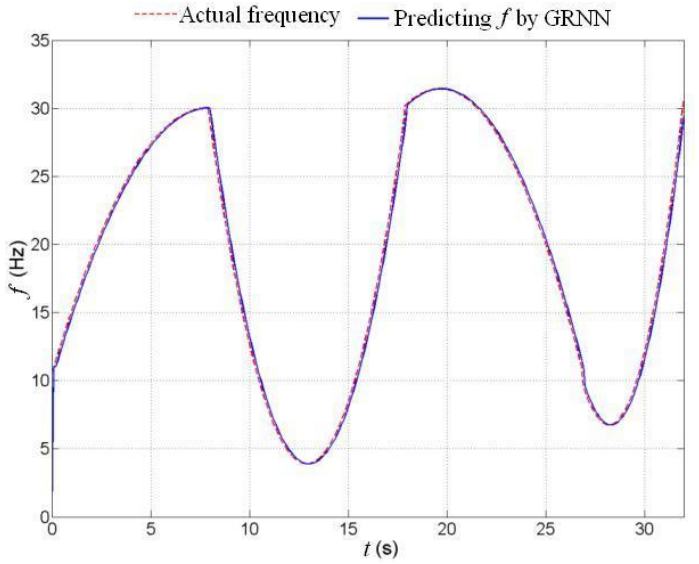

(a) Two polynomial signal identification

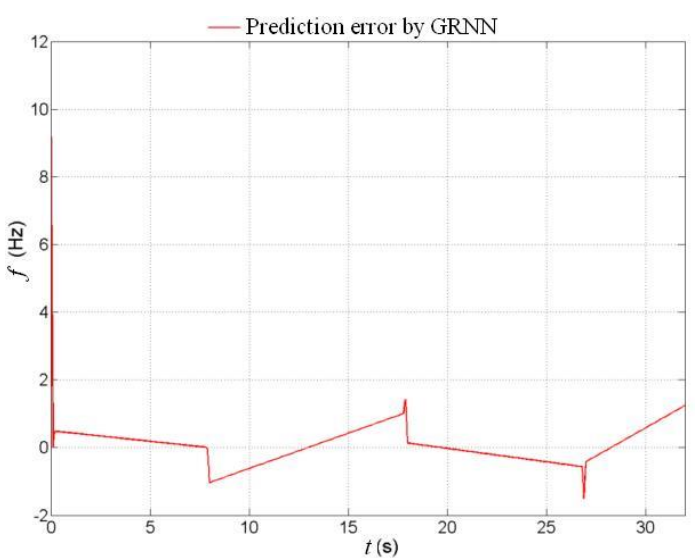

(b) Identification error

\section{Figure 7. Resonance frequency identification results and error by GRNN}

The second order polynomial curve is used to verify the prediction accuracy of GRNN because the resonant frequency has a quadric correlation with the drilling depth in drilling experiments. The newest 16 sets of data are used to train the GRNN that mentioned above to predict the 17th frequency. The effect of the simulation is quite ideal and the prediction result is shown in Figure 7. All the simulations are programmed with Matlab version 7.1, and run on a Pentium 4 with a clock speed of $2.5 \mathrm{GHz}$ and 1 GB of RAM under a Microsoft Windows XP environment. 


\subsection{Frequency adaptive control based on ANFIS}

In this paper, ANFIS (Adaptive Neural Fuzzy Inference System) control strategy that used for vibration frequency control has 3 input variables (resonance amplitude $A_{\max }(t)$, variety of amplitude $d A_{\max }$, frequency prediction error ef $\left.(t)=f_{o}(t)-\hat{f}(t)\right)$ and 1 output variable (swept frequency bandwidth, $\Delta f$ ) which structure is as shown in Fig.8. The typical architecture of ANFIS that used is Takagi-Sugeno (T-S type) fuzzy models consisting of five layers, and every layer has the node as shown in Fig.8. It uses a hybrid learning algorithm, which utilizes the capability of fuzzy and neural network. The ANFIS with 3 input variables and 1 output variable needs 125 fuzzy control rules because each variable can be divided into 5 levels, namely, negative big (NB), negative small (NS), zero (ZE), positive small (PS), and positive big(PB). The output variable (the swept frequency width) is a linear function of the three input variables. The rulebase contains 125 fuzzy IF-THEN rules of TS type as follows:

$$
\text { Rule } i \text { : If } A \text { is } X \text {, and } d A \text { is } Y \text {, and } e f \text { is } Z \text {, then } f_{i}=p_{i} A+q_{i} d A+r_{i} e f+s_{i}
$$

Where $X, Y$, and $Z$ are particular fuzzy subsets defined by nonlinear coefficient. And $f_{i}$ is an exciting function, while $p_{i}, q_{i}, r_{i}$, and $s_{i}$ are the linear coefficients determining the output of each applied fuzzy rule and usually known as consequent parameters.

ANFIS has 5 layers [18], as shown in Figure 8. The function of each layer is as follow:

Layer 1-translates the input variables into fuzzy variables. Each node in this layer is an adaptive node with a node activation function parameter. The outputs of the layer are: $\mu^{N B}\left(A_{k}\right), \mu^{N S}\left(A_{k}\right), \mu^{Z E}\left(A_{k}\right), \mu^{P S}\left(A_{k}\right), \mu^{P B}\left(A_{k}\right), \mu^{N B}\left(d A_{k}\right), \ldots \ldots, \mu^{P B}\left(e f_{k}\right)$. The membership function is given by the generalized Gaussian function as shown below:

$$
\mu_{i}(x)=\exp \left[-\left(\frac{x-c_{i}}{\sigma_{i}}\right)\right]
$$

Where $\left\{c_{i}, \sigma_{i}\right\}$ are adaptive parameters of the node. The number of the nodes in this layer is 15 and the parameters in this layer are referred to as premise parameters.

Layer 2-implementation the calculation of the condition's section fuzzy set, output the fitness of each rule. Every node in this layer is a fixed node whose output is the product of all the incoming signals. The output of the nodes in the layer are just similar to $\omega_{1}=\mu^{P B}\left(A_{k}\right) \cdot \mu^{P B}\left(d A_{k}\right) \cdot \mu^{P B}\left(e f_{k}\right), \ldots \ldots, \omega_{125}=\mu^{N B}\left(A_{k}\right) \cdot \mu^{N B}\left(d A_{k}\right) \cdot \mu^{N B}\left(e f_{k}\right)$.

Layer 3 -normalizes the fitness of each rule. The $i$ th node calculates the ratio of the gain ratio $i$ th rule incentive strength to the sum of that of all rules and can be presented as $\bar{\omega}_{i}=\omega_{i} / \sum_{j=1}^{125} \omega_{j}$.

Layer 4-Every node in this layer is an adaptive node which can calculate the output of each rule with the function as $u_{i}=\bar{\omega}_{i} f_{i}=\bar{\omega}_{i}\left(p_{i} A+q_{i} d A+r_{i} e f+s_{i}\right)(i=1,2, \cdots, 125)$. Where $\bar{\omega}_{i}$ is a normalized incentive strength from layer 3 and $\left\{p_{i}, q_{i}, r_{i}, s_{i}\right\}$ is the parameter set of the node. Parameters in this layer are referred to as consequent parameters. 


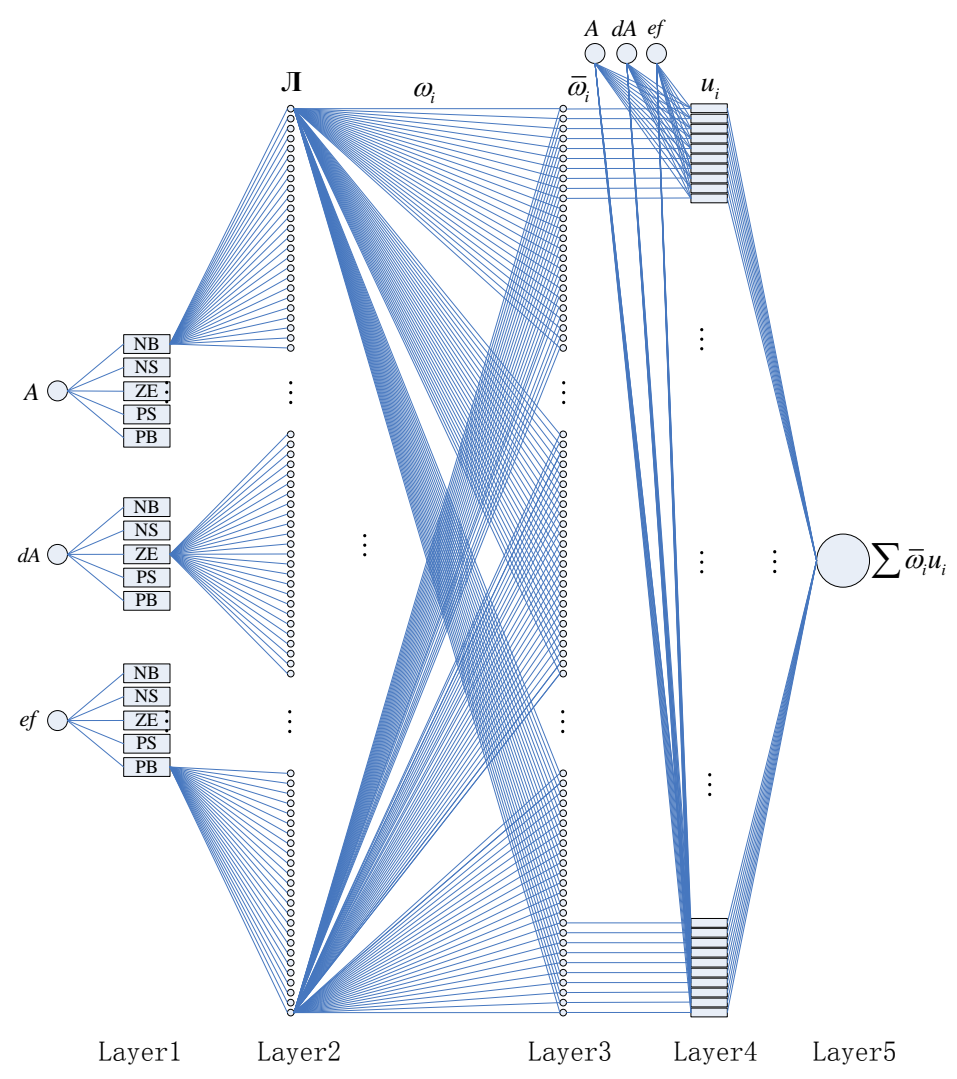

Figure 8. Network configuration of ANFIS

Layer 5-the single node in this layer is a fixed node which calculates all the input signals as the output, such as $\delta_{i}(k)=\sum_{i=1}^{125} \bar{\omega}_{i} f_{i}$.

The input variables of the dynamic swept frequency ANFIS are resonance amplitude $A_{\max }(t)$, variety of amplitude $d A_{\max }$, and frequency prediction error $e f(t)=f_{o}(t)-\hat{f}(t)$. And the resonance amplitude $A \in[0,1](\mathrm{mm})$, variety of amplitude $d A \in[-3,3](\mathrm{mm} / \mathrm{s})$, frequency prediction error $e f \in[0,5](\mathrm{Hz})$, and the output swept frequency width $\Delta f \in[0,20](\mathrm{Hz})$. The input and output domains of the controller are $A^{\prime} \in[0,1], d A^{\prime} \in[-1,1], e f^{\prime} \in[0,1]$, and $\Delta f \in[0,1]$ respectively. The quantifying factors $k_{A}=1, k_{d A}=1 / 3$ and the scaling factor $k_{f}=20$.

The experience curves between the sampling head's vibration amplitude, variety of amplitude, frequency prediction error and swept frequency width are shown in Fig.9 by our drilling experiments, where the fuzzy domains of input variables are as $\mathrm{X}$-axis and the swept frequency width is as the Y-axis. So we can obtain 18081 sets of empirical data by the curves for training the ANFIS controller. 


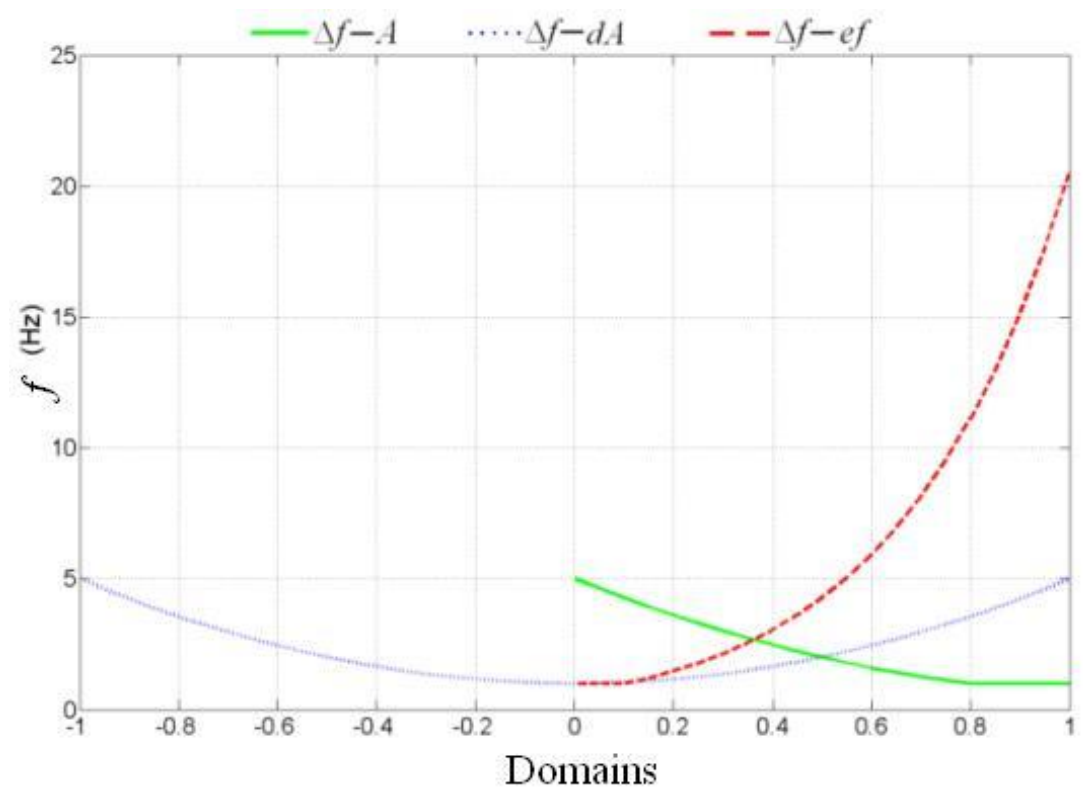

Figure 9. The experience curve between the inputs and sweep frequency

ANFIS controller is simulated using 18081 sets of empirical data that mentioned above by MATLAB7.1. And 125 fuzzy rules are obtained after $215 \mathrm{~s}$ training and the error is $1.57767 \times 10^{-3} \mathrm{~Hz}$. The control surface figures of ANFIS after training are as shown in Figure 10.
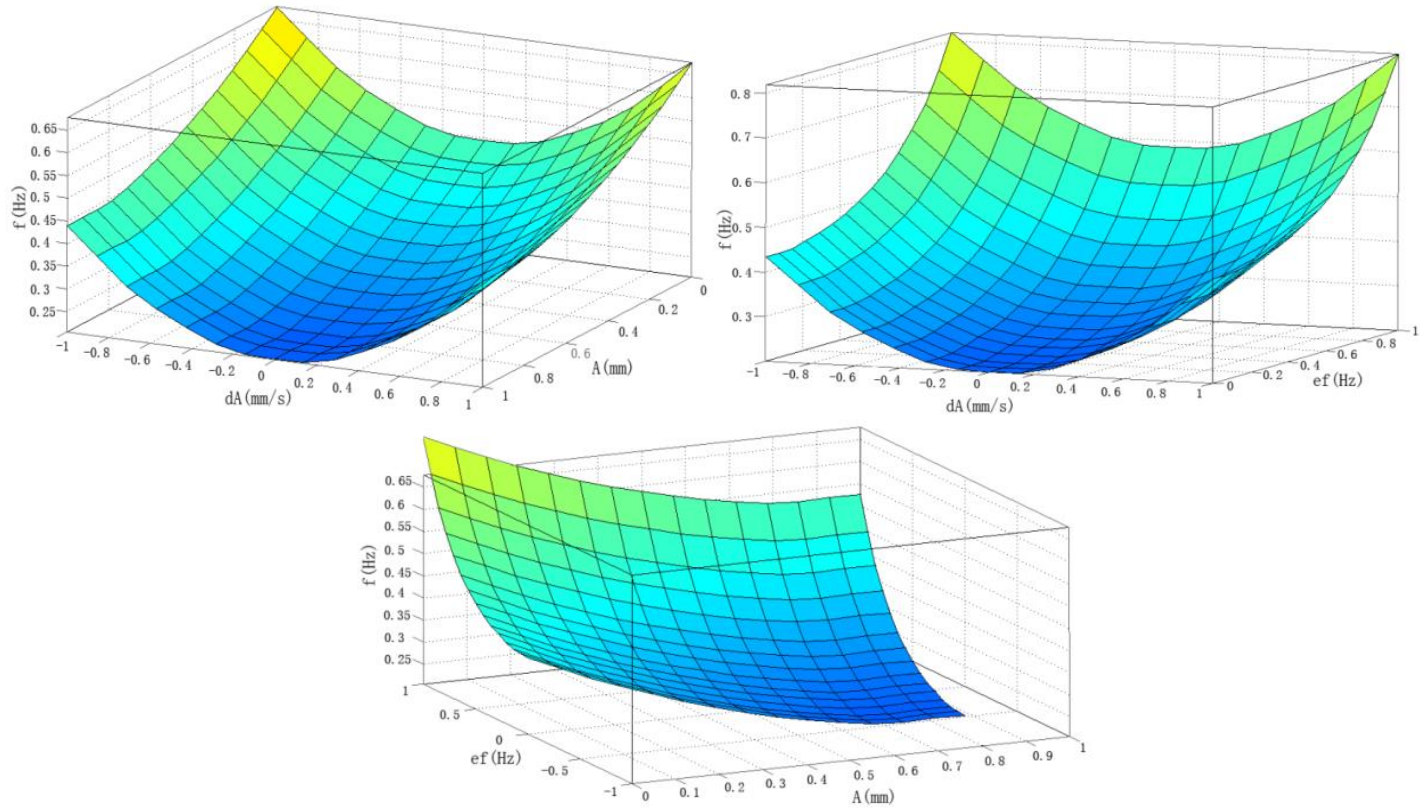

Figure 10. Output surface figure of ANFIS 


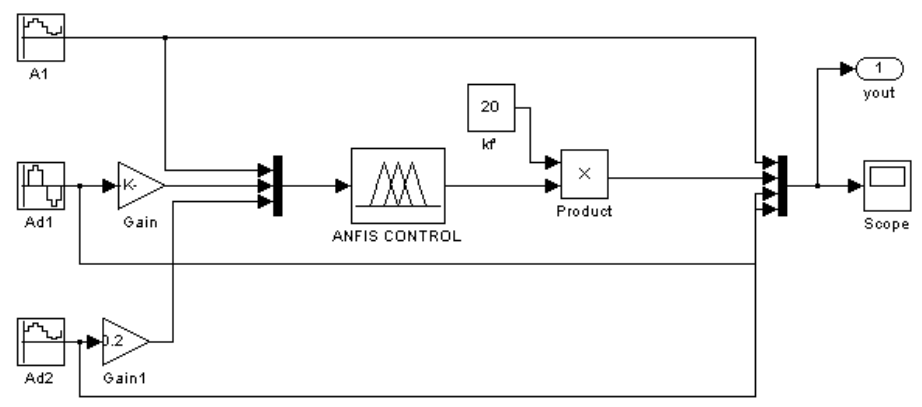

Figure 11. Simulink figure of the sweep frequency neural-fuzzy adaptive control

In order to verify the validity of the ANFIS controller after training, the performance is simulated using MATLAB7 Simulink program, as shown in Figure 11.

The signals that used for simulation are as follows: the sine signal is used as the vibration amplitude signal with the range of $[0,1]$, the sine signal and the saw tooth signal are used as the variety of vibration amplitude and frequency prediction error respectively with the range of $[-3,3]$ and $[0,5]$. Figure 12 shows the simulation results.
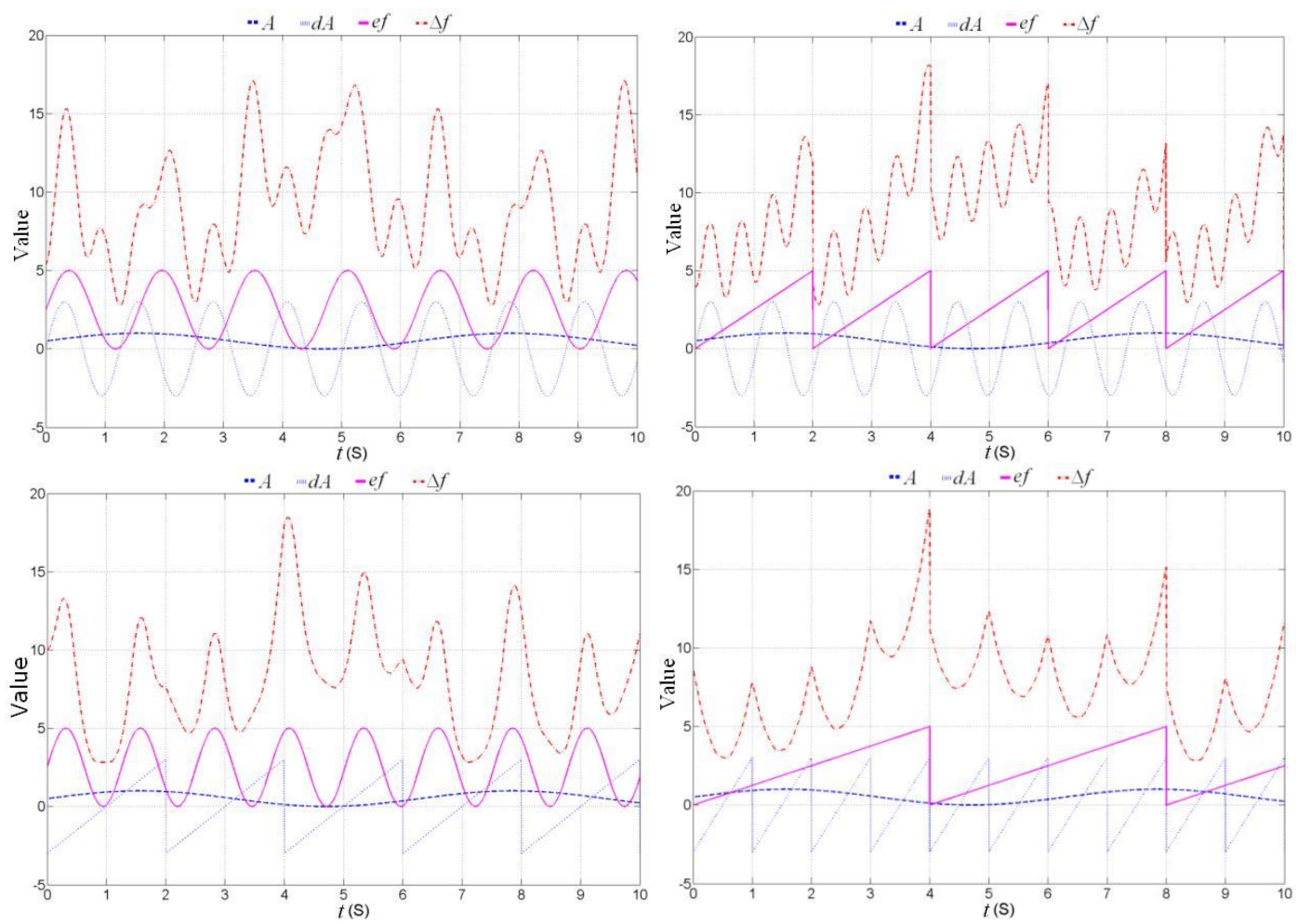

Figure 12. Simulation results of the sweep frequency ANFIS

As Figure 12 shows, the ANFIS controller that mentioned above can adjust the frequency width effectively according to the changes of input signals. It can improve the sweep efficiency and real-time control when vibration amplitude of sampling head is large and other two input parameters are small. It can also expand the search range of the resonant frequency automatically to insure that the frequency sweep range can cover 
the new resonant frequency when the input signals are in the opposite situation. So, the ANFIS controller can meet the needs of the swept frequency width adaptive control in virtue of its excellent dynamic performance and high precision.

\section{Experimental Results and Discussion}

Sampling experiments have been done in the simulant lunar soil with and without ANFIS control strategy respectively. The drilling depth with and without adaptive control are compared in the Figure 13. The predicted frequency $\hat{f}$ by using GRNN, the swept frequency width $\Delta f$ using ANFIS are shown in Figure 14.

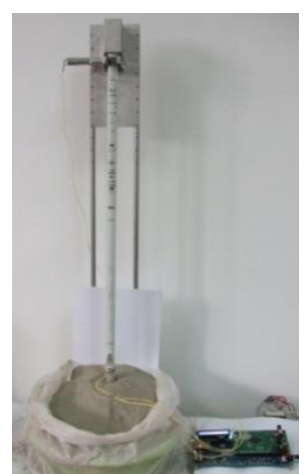

(a) No vibration

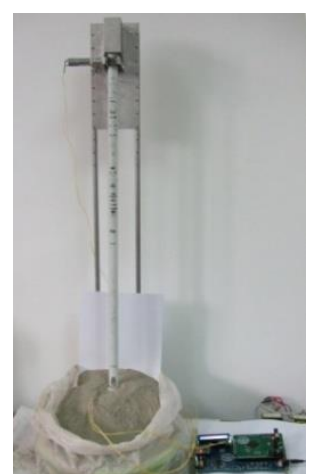

(b) With vibration

Figure 13. Contrast sampling experiments in stimulant lunar regolith with and without vibration

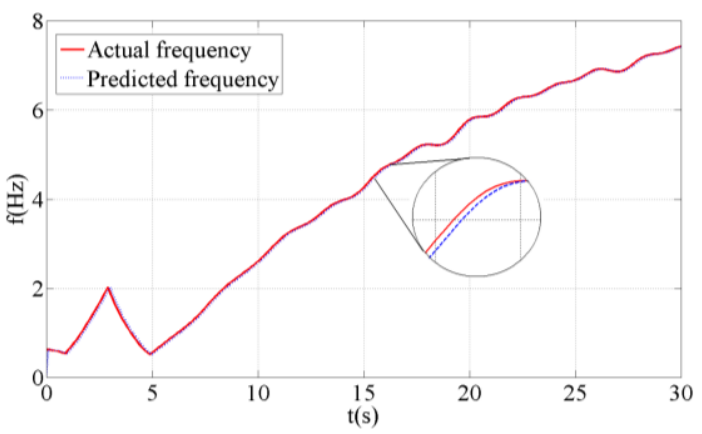

(a) Frequency prediction

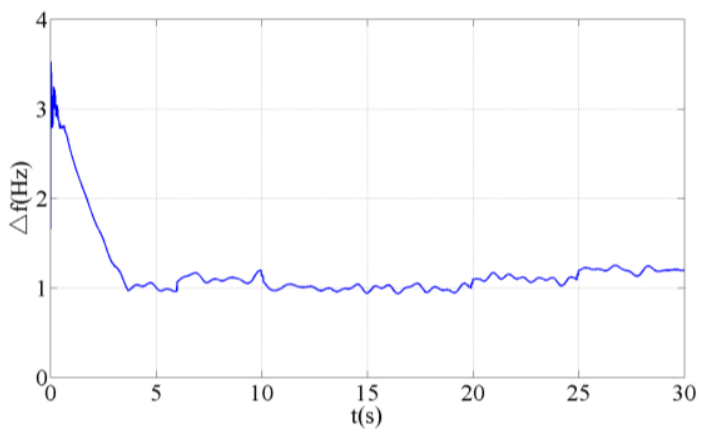

(b) Sweep frequency band

\section{Figure 14. Experimental results of the vibration frequency ANFIS control based on the dynamic frequency prediction by GRNN}

The experimental results show that the value of the swept frequency width $\Delta f$ is large in the beginning owing to the large prediction error $e f$. With the increasing number of data, frequency prediction tends to be more accurate and the swept frequency width decreases sharply. The vibration system can work in the narrow-band swept frequency condition and track the dynamic "sampling head - regolith" resonant frequency when it reaches a stable working state. What's more, the drilling depth, drilling speed and the time of discarding the sample is $97 \mathrm{~mm}, 3 \mathrm{~s}$ and $13 \mathrm{~s}$ respectively when the sampling head 
keeps vibrating without adaptive control. A compared experimental result shows that the drilling depth, drilling speed and discarding time is $115 \mathrm{~mm}, 3.8 \mathrm{~s}$ and $7.5 \mathrm{~s}$ respectively when the vibration of the sampling head is under the vibration swept frequency ANFIS control based on the dynamic prediction by GRNN that mentioned above. What's more, the drilling depth, drilling speed and discarding time is $69 \mathrm{~mm}$, $2.3 \mathrm{~s}$ and $23 \mathrm{~s}$ respectively without vibration. The compared experimental results are shown in Table 1. Additionally, the experimental results of the same comparison in cement are shown in Table 2.

\section{Table 1. Comparison of the experimental results in stimulant lunar regolith}

\begin{tabular}{|c|c|c|c|}
\hline & No vibration & $\begin{array}{c}\text { Vibration } \\
\text { without } \\
\text { control }\end{array}$ & $\begin{array}{c}\text { Vibration frequency } \\
\text { adaptive control }\end{array}$ \\
\hline Drilling depth (mm) & 69 & 97 & 115 \\
\hline $\begin{array}{c}\text { Drilling speed } \\
\text { (mm/s) }\end{array}$ & 2.3 & 3 & 3.8 \\
\hline Discarding time (s) & 23 & 13 & 7.5 \\
\hline
\end{tabular}

Table 2. Comparison of the experimental results in cement

\begin{tabular}{|c|c|c|c|}
\hline & No vibration & $\begin{array}{c}\text { Vibration without } \\
\text { control }\end{array}$ & $\begin{array}{c}\text { Vibration frequency } \\
\text { adaptive control }\end{array}$ \\
\hline Drilling depth (mm) & 65 & 90 & 105 \\
\hline $\begin{array}{c}\text { Drilling speed } \\
\text { (mm/s) }\end{array}$ & 2.2 & 2.8 & 3.7 \\
\hline Discarding time (s) & 25 & 14 & 8.5 \\
\hline
\end{tabular}

\section{Conclusions}

1) In the paper, a novel flexible sampling robot used for sampling the shallow lunar regolith is developed. It can expand not less than $1 \mathrm{~m}$ and can meet the need of shallow lunar regolith sampling. The "sampling head - lunar regolith" resonant broking system was built and studied by Runge-Kutta method and it draws the conclusion that the sampler in the resonant state is most helpful to drilling.

2) The vibration swept frequency ANFIS control system based on the dynamic prediction by using GRNN was proposed. The simulation results verified the feasibility of the algorithm. Finally, experimental results show that the proposed control strategy demonstrates high efficient drilling in the simulant lunar regolith and cement. More specifically, it can improve the drilling depth by $66.7 \%$ and $61.5 \%$, improve the drilling speed by about $65.2 \%$ and $68.2 \%$, improve the efficiency by $67.4 \%$ and $66 \%$ in stimulant lunar regolith and cement, respectively.

\section{Acknowledgements}

This work is supported by the Natural Science Foundation of Jiangsu Province (BK20130696), the Jiangsu Planned Projects for Postdoctoral Research Funds(1202005C) and the Natural Science Foundation of China (No. 61105048, No. 61104206, No. 61075068). The authors are also grateful for the State Key Laboratory of Environmental Geochemistry, Institute of Geochemistry, Chinese Academy of Sciences for providing a simulated lunar regolith (model CAS-1). 


\section{References}

[1] Z. Y. Ouyang, "International lunar exploration progress and Chinese lunar exploration", J. Geological Science and Technology Information, vol. 23, no. 4, (2004), pp. 1-5.

[2] L. L. Kashkavov, L. I. Genayeva and A. K. Lavrukhina, "Radiation history of matter returned by the Soviet automatic stations Lunar-16 and Lunar-20, according to data of track investigations", NASA-75N-75523[R] Washington: NASA, (1974).

[3] NASA JPL, Spacecraft: surface operations: instruments: rock abrasion tool [R/OL]", (2012) February 7, http://marsrovers.jpl.nasa.gov/mission/spacecraft_instru_rat.html.

[4] R. Fleischner, "Concurrent Actuator Development for the Mars Exploration Instrument Deployment Device", Proceedings of the 10th European Space Mechanisms and Tribology Symposium, San Sebastián, Spain, (2003) September 24-26.

[5] C. R. Stoker, L. Richter and W. H. Smith, "The mars underground mole (MUM): A subsurface penetration device with in situ infrared reflectance and Raman spectroscopic sensing capability", Proceedings of the $6^{\text {소 }}$ International Conference on Mars, Pasadena, California, (2003) July 20-25.

[6] T. Kubota, I. Nakatani and K. Watanabe, "Study on Mole-Typed Deep Driller Robot for Subsurface Exploration", Proceedings of the 2005 IEEE International Conference on Robotics and Automation, Barcelona, Spain, (2005) April 18-22.

[7] T. Kubota, K. Watanabe and S. Tanaka, "Earth-worm typed drilling robot for subsurface planetary exploration", Proceedings of the 2007 IEEE International Conference on Robotics and Biomimetics, Sanya, China, (2007) December 15-18.

[8] Y. Gao, A. Ellery and M. Jaddou, "Planetary Micro-Penetrator Concept Study with Biomimetric Drill and Sampler Design", J. IEEE Transactions on Aerospace and Electronic Systems, vol. 43, no. 3, (2007), pp. 875885.

[9] N. I. Kömlea, E. Kaufmanna and G. Kargla, "Development of thermal sensors and drilling systems for lunar and planetary regoliths", J. Advances in Space Research, vol. 42, no. 2, (2008), pp. 363-368.

[10] K. Hu, A. G. Song and W. L. Wang, "Fault Detection and Estimation for Non-Gaussian Stochastic Systems with Time Varying Delay”, J. Advances in Difference Equations, vol. 1, no. 22, (2013).

[11] F. A. Shirazi, J. Monhammadpour, K. M. Grigoriadis and S. Gangbing, "Identification and control of an MR damper with stiction effect and its application in structural vibration mitigation", J. IEEE Transanctions on Control Systems Technology, vol. 20, no. 5, (2012), pp. 1285-1301.

[12] C. Spelta,F. Previdi and S. M. Savaresi, "Control of magnetorheological dampers for vibration reduction in a washing machine", J. Mechatronics, vol. 19, (2009), pp. 410-421.

[13] D. Q.Truong, Q. T. Truong and K. A. Kyoung, "Development of a novel linear magnetic actuator with trajectory control based on an online tuning fuzzy PID controller", J. International Journal of Precision Engineering and Manufacturing, vol. 13, no. 8, (2012), pp. 1403-1411.

[14] J. Lin and Y. B. Zheng, "Vibration suppression control of smart piezoelectric rotating truss structure by parallel neuro-fuzzy control with genetic algorithm tuning", J. Journal of Sound and Vibration, vol. 331, no. 16, (2012), pp. 3677-3694.

[15] N. Kumar and S. P. Singh, "Vibration control of curved panel using smart damping", J. Mechanical Systems and Signal Processing, vol. 30, (2012), pp. 232-247.

[16] W. Lu, Y. Ling and A. G. Song, "Measuring tape-like sampling arm and drill for sampling lunar regolith", J. Int J Adv Robotic Sy, vol. 10, (2013), pp. 1-9.

[17] S. B. Yao, D. L. Guo and G. W. Yang, "Three-dimensional aerodynamic optimization design of high-speed train nose based on GA-GRNN", J. Science China, vol. 55, no. 11, (2012), pp. 3118-3130.

[18] P. H. Hidayat and S. Sasongko, "Performance analysis of adaptive neuro fuzzy inference systems (ANFIS) for speed control of brushless DC motor", Proceeding of the 2011 International Conference on Electrical Engineering and Informatics, Indonesia, Bandung, (2011) July 17-19. 
International Journal of Control and Automation Vol.7, No.3 (2014) 